\title{
A RANDOM PERMUTATION MODEL ARISING IN CHEMISTRY
}

\author{
MARK BROWN,* The City College of New York \\ EROL A. PEKÖZ,** Boston University \\ SHELDON M. ROSS, ${ }^{* *}$ University of Southern California
}

\begin{abstract}
We study a model arising in chemistry where $n$ elements numbered $1,2, \ldots, n$ are randomly permuted and if $i$ is immediately to the left of $i+1$ then they become stuck together to form a cluster. The resulting clusters are then numbered and considered as elements, and this process keeps repeating until only a single cluster is remaining. In this article we study properties of the distribution of the number of permutations required.
\end{abstract}

Keywords: Random permutation; hat-check problem

2000 Mathematics Subject Classification: Primary 60C05

Secondary $92 \mathrm{E} 20$

\section{Introduction}

For the classic hat-check problem first proposed in 1708 by Montmort [2], the following variation appears in [6, p. 93]. Each member of a group of $n$ individuals throws his or her hat in a pile. The hats are shuffled, each person chooses a random hat, and the people who receive their own hat depart. Then the process repeats with the remaining people until everybody has departed; let $N$ be the number of shuffles required. With $X_{i}$ representing the total number of people who have departed after shuffle number $i$, it is easy to show that $X_{i}-i$ is a martingale and, thus, by the optional sampling theorem we elegantly see that $\mathrm{E}[N]=n$.

Someone getting their own hat can also be thought of as corresponding to a cycle of length one in a random permutation. Properties of cycles of various lengths in random permutations have been studied extensively; see [1] and [3] for entry points to this literature. A variation of this problem was presented in [5], where it was given as a model for a chemical bonding process. Below we discuss this variation and study its properties. We quote the following description of the chemistry application from [5], where a recursive formula was given to numerically compute the mean.

There are 10 molecules in some hierarchical order operating in a system. A catalyst is added to the system and a chemical reaction sets in. The molecules line up. In the line-up from left to right molecules in consecutive increasing hierarchical order bond together and become one. A new hierarchical order sets among the fused molecules. The catalyst is added again

\footnotetext{
Received 30 September 2008; revision received 7 November 2008.

* Postal address: Department of Mathematics, The City College of New York, New York, NY 10031-9100, USA.

Email address: cybergarf@aol.com

Supported by the National Security Agency under grant H98230-06-01-0149.

** Postal address: Department of Operations and Technology Management, Boston University, 595 Commonwealth Avenue, Boston, MA 02215, USA. Email address: pekoz@bu.edu

*** Postal address: Department of Industrial and System Engineering, University of Southern California, Los Angeles, CA 90089, USA. Email address: smross@usc.edu
} 
to the system and the whole process starts all over again. The question raised is how many times catalysts are expected to be added in order to get a single lump of all molecules.

This variation presented in [5] can be abstractly stated as follows. Suppose that we have $n$ elements numbered $1,2, \ldots, n$. These elements are randomly permuted, and if $i$ is immediately to the left of $i+1$ then $i$ and $i+1$ become stuck together to form (possibly with other adjacently numbered elements) a cluster. These clusters are then randomly permuted and if a cluster ending with $i$ immediately precedes one starting with $i+1$ then those two clusters join together to form a new cluster. This continues until there is only one cluster, and we are interested in $N(n)$, the number of permutations that are needed. For instance, suppose that $n=7$ and that the first permutation is

$$
3,4,5,1,2,7,6 \text {, }
$$

which results in the clusters $\{3,4,5\},\{1,2\},\{6\}$, and $\{7\}$. If a random permutation of these four clusters gives the ordering

$$
\{6\},\{7\},\{3,4,5\},\{1,2\}
$$

then the new sets of clusters are $\{6,7\},\{3,4,5\}$, and $\{1,2\}$. If a random permutation of these three clusters gives the ordering

$$
\{3,4,5\},\{6,7\},\{1,2\}
$$

then the new sets of clusters are $\{3,4,5,6,7\}$ and $\{1,2\}$. If a random permutation of these two clusters gives the ordering

$$
\{1,2\},\{3,4,5,6,7\}
$$

then there is now a single cluster $\{1,2,3,4,5,6,7\}$ and $N(7)=4$.

The random variable $N(n)$ can be analyzed as a first passage time from state $n$ to state 1 of a Markov chain whose state is the current number of clusters. When the state of this chain is $i$, we will designate the clusters as $1, \ldots, i$, with 1 being the cluster whose elements are smallest, 2 being the cluster whose elements are the next smallest, and so on. For instance, in the preceding $n=7$ case, the state after the first transition is 4 , with 1 being the cluster $\{1,2\}, 2$ being the cluster $\{3,4,5\}, 3$ being the cluster $\{6\}$, and 4 being the cluster $\{7\}$. With this convention, the transitions from state $i$ are exactly the same as if the problem began with the $i$ elements, $1, \ldots, i$.

In Section 2 we compute the transition probabilities of this Markov chain and use them to obtain some stochastic inequalities. In Section 3 we obtain upper and lower bounds on $\mathrm{E}[N(n)]$, as well as bounds on its distribution. In Section 4 we give results for a circular version of the problem.

\section{The transition probabilities}

With the above definitions, let $D_{n}$ be the decrease in the number of clusters starting from state $n$. Then we have the following proposition.

Proposition 1. For $0 \leq k<n$,

$$
\mathrm{P}\left(D_{n}=k\right)=\frac{n-k+1}{n k !} \sum_{i=0}^{n-k+1} \frac{(-1)^{i}}{i !} .
$$


Proof. Letting $A_{i}$ be the event that $i$ immediately precedes $i+1$ in the random permutation, then $D_{n}$ is the number of events $A_{1}, \ldots, A_{n-1}$ that occur. Then, with

$$
S_{j}=\sum_{0<i_{1}<\cdots<i_{j}<n} \mathrm{P}\left(A_{i_{1}} \cdots A_{i_{j}}\right),
$$

the inclusion/exclusion identity (see [4, p. 106]) gives

$$
\mathrm{P}\left(D_{n}=k\right)=\sum_{j=k}^{n-1} S_{j}\left(\begin{array}{l}
j \\
k
\end{array}\right)(-1)^{j+k} .
$$

Now consider $\mathrm{P}\left(A_{i_{1}} \cdots A_{i_{j}}\right)$. If we think of a permutation of $n$ elements as having $n$ degrees of freedom then, for each event $A_{i}$ in the intersection, one degree of freedom in the permutation is dropped. For instance, suppose that we want $\mathrm{P}\left(A_{2} A_{3} A_{6}\right)$. Then, in order for these three events to occur, 2, 3, and 4 must be consecutive values of the permutation, as must be 6 and 7 . Because there are $n-5$ other values, there are thus $(n-3)$ ! such permutations. Similarly, for the event $A_{2} A_{4} A_{6}$ to occur, 2 and 3 must be consecutive values of the permutation, as must be 4,5 and 6,7 . As there are $n-6$ other values, there are $(n-3)$ ! such permutations. Consequently, for $0<i_{1}<\cdots<i_{j}<n$,

As a result,

$$
\mathrm{P}\left(A_{i_{1}} \cdots A_{i_{j}}\right)=\frac{(n-j) !}{n !} .
$$

$$
S_{j}=\left(\begin{array}{c}
n-1 \\
j
\end{array}\right) \frac{(n-j) !}{n !}=\frac{n-j}{n j !}
$$

which yields

$$
\begin{aligned}
\mathrm{P}\left(D_{n}=k\right) & =\sum_{j=k}^{n-1}\left(\begin{array}{l}
j \\
k
\end{array}\right)(-1)^{j+k} \frac{n-j}{n j !} \\
& =\sum_{i=0}^{n-k-1}(-1)^{i}\left(\begin{array}{c}
k+i \\
k
\end{array}\right) \frac{n-k-i}{n(k+i) !} \\
& =\sum_{i=0}^{n-k-1}(-1)^{i} \frac{n-k-i}{n k ! i !} \\
& =\frac{1}{n k !}\left((n-k) \sum_{i=0}^{n-k-1} \frac{(-1)^{i}}{i !}-\sum_{i=1}^{n-k-1} \frac{(-1)^{i}}{(i-1) !}\right) \\
& =\frac{1}{n k !}\left((n-k+1) \sum_{i=1}^{n-k-2} \frac{(-1)^{i}}{i !}+(n-k) \frac{(-1)^{n-k-1}}{(n-k-1) !}\right) .
\end{aligned}
$$

Thus, the result follows once we show that

$$
(n-k) \frac{(-1)^{n-k-1}}{(n-k-1) !}=(n-k+1)\left(\frac{(-1)^{n-k-1}}{(n-k-1) !}+\frac{(-1)^{n-k}}{(n-k) !}+\frac{(-1)^{n-k+1}}{(n-k+1) !}\right)
$$

or, equivalently, that

$$
\frac{(-1)^{n-k}}{(n-k-1) !}=(n-k+1)\left(\frac{(-1)^{n-k}}{(n-k) !}+\frac{(-1)^{n-k+1}}{(n-k+1) !}\right)
$$


or

$$
1=(n-k+1)\left(\frac{1}{n-k}-\frac{1}{(n-k+1)(n-k)}\right),
$$

which is immediate.

Remark 1. A recursive expression for $\mathrm{P}\left(D_{n}=k\right)$, though not in closed form, was given in [5].

From Proposition 1 we immediately conclude that $D_{n}$ converges in distribution to a Poisson random variable with mean 1.

Corollary 1. We have $\lim _{n \rightarrow \infty} \mathrm{P}\left(D_{n}=k\right)=\mathrm{e}^{-1} / k$ !

We now present two results that will be used in the next section. Recall from [6, p. 133] that a discrete random variable $X$ is said to be likelihood ratio smaller than $Y$ if $\mathrm{P}(X=k) / \mathrm{P}(Y=k)$ is nonincreasing in $k$.

Corollary 2. With the above definitions, $D_{n}$ is likelihood ratio smaller than a Poisson random variable with mean 1.

Proof. We need to show that $k ! \mathrm{P}\left(D_{n}=k\right)$ is nonincreasing in $k$. But, with $B_{k}=$ $n k ! \mathrm{P}\left(D_{n}=k\right)$ we have

$$
\begin{aligned}
B_{k-1}-B_{k} & =\sum_{i=0}^{n-k+1} \frac{(-1)^{i}}{i !}+(n-k+2) \frac{(-1)^{n-k+2}}{(n-k+2) !} \\
& =\sum_{i=0}^{n-k} \frac{(-1)^{i}}{i !} \\
& >0
\end{aligned}
$$

which proves the result.

Corollary 3. The state of the Markov chain after a transition from state $n, n-D_{n}$, is likelihood ratio increasing in $n$.

Proof. From Proposition 1,

$$
\mathrm{P}\left(n-D_{n}=k\right)=\frac{k+1}{n(n-k) !} \sum_{i=0}^{k+1} \frac{(-1)^{i}}{i !} .
$$

Consequently,

$$
\frac{\mathrm{P}\left(n+1-D_{n+1}=k\right)}{\mathrm{P}\left(n-D_{n}=k\right)}=\frac{n}{(n+1)(n+1-k)} .
$$

As the preceding is increasing in $k$, the result follows.

\section{The random variable $N(n)$}

Let $X_{i}$ be the $i$ th decrease in the number of clusters, so that

$$
S_{k} \equiv n-\sum_{i=1}^{k} X_{i}
$$

is the state of the Markov chain, starting in state $n$, after $k$ transitions, $k \geq 1$. 
Proposition 2. We have

$$
\mathrm{P}(N(n)>k) \geq \sum_{i=0}^{n-1} \frac{\mathrm{e}^{-k} k^{i}}{i !} .
$$

Proof. Let the $Y_{i}, i=1, \ldots, k$, be independent Poisson random variables, each with mean 1. Now, because likelihood ratio is a stronger ordering than stochastic order (see Proposition 4.20 of [6]), it follows by Corollary 2 that $X_{i}$, conditional on $X_{1}, \ldots, X_{i-1}$, is stochastically smaller than a Poisson random variable with mean 1 . Consequently, the random vector $X_{1}, \ldots, X_{k}$ can be generated in such a manner that $X_{i} \leq Y_{i}$ for each $i=1, \ldots, k$. But this implies that

$$
\begin{aligned}
\mathrm{P}(N(n)>k) & =\mathrm{P}\left(X_{1}+\cdots+X_{k}<n\right) \\
& \geq \mathrm{P}\left(Y_{1}+\cdots+Y_{k}<n\right) \\
& =\sum_{i=0}^{n-1} \frac{\mathrm{e}^{-k} k^{i}}{i !} .
\end{aligned}
$$

We now consider bounds on $\mathrm{E}[N(n)]$.

Proposition 3. We have

$$
\mathrm{E}[N(n)] \leq n-1+\sum_{i=1}^{n-1} \frac{1}{i}
$$

Proof. First note that

$$
\mathrm{E}\left[D_{n}\right]=\sum_{i=1}^{n-1} \mathrm{P}(i \text { immediately precedes } i+1)=\frac{n-1}{n} .
$$

Because the Markov chain cannot make a transition from a state into a higher state and $\mathrm{E}\left[D_{n}\right]$ is nondecreasing in $n$, it follows from Proposition 5.23 of [6] that

$$
\mathrm{E}[N(n)] \leq \sum_{i=2}^{n} \frac{1}{\mathrm{E}\left[D_{i}\right]}=n-1+\sum_{i=1}^{n-1} \frac{1}{i}
$$

Proposition 4. We have

$$
\mathrm{E}[N(n)] \geq n-1+\frac{\mathrm{e}}{n(\mathrm{e}-1)}+\frac{\mathrm{e}}{(\mathrm{e}-1)^{2}} \sum_{j=2}^{n-1} \frac{1}{j} .
$$

Proof. To begin, note that

$$
Z_{k}=\sum_{i=1}^{k}\left(X_{i}-\mathrm{E}\left[X_{i} \mid X_{1}, \ldots, X_{i-1}\right]\right), \quad k \geq 1,
$$

is a zero-mean martingale. Hence, by the martingale stopping theorem,

$$
\mathrm{E}\left[Z_{N(n)}\right]=0 .
$$


Now, because $\mathrm{E}\left[X_{i} \mid X_{1}, \ldots, X_{i-1}\right]$ is the expected decrease from state $S_{i-1}$, it follows from (1) that

$$
\mathrm{E}\left[X_{i} \mid X_{1}, \ldots, X_{i-1}\right]=\mathrm{E}\left[X_{i} \mid S_{i-1}\right]=\mathrm{E}\left[D_{S_{i-1}} \mid S_{i-1}\right]=1-\frac{1}{S_{i-1}} .
$$

Using this, and the fact that $\sum_{i=1}^{N(n)} X_{i}=n-1$, we obtain, from (2) and (3),

$$
n-1-\mathrm{E}[N(n)]+\mathrm{E}\left[\sum_{i=1}^{N(n)} \frac{1}{S_{i-1}}\right]=0
$$

Now (notationally suppressing its dependence on the initial state $n$ ), let $T_{j}$ denote the amount of time that the Markov chain spends in state $j, j>1$. Then

$$
\sum_{i=1}^{N(n)} \frac{1}{S_{i-1}}=\sum_{j=2}^{n} \frac{T_{j}}{j}
$$

Hence,

$$
\mathrm{E}[N(n)]=n-1+\sum_{j=2}^{n} \frac{1}{j} \mathrm{E}\left[T_{j}\right] \geq n-1+\frac{\mathrm{e}}{(\mathrm{e}-1)^{2}} \sum_{j=2}^{n-1} \frac{1}{j}+\frac{\mathrm{e}}{n(\mathrm{e}-1)},
$$

where, for the inequality, we made use of the following proposition.

Proposition 5. We have

$$
\begin{aligned}
& \mathrm{E}\left[T_{n}\right]=\frac{1}{\mathrm{P}\left(D_{n}>0\right)} \geq \frac{\mathrm{e}}{\mathrm{e}-1}, \\
& \mathrm{E}\left[T_{j}\right]=\frac{\mathrm{P}\left(T_{j}>0\right)}{\mathrm{P}\left(D_{j}>0\right)} \geq \frac{\mathrm{e}}{(\mathrm{e}-1)^{2}} .
\end{aligned}
$$

To prove Proposition 5, we will need a series of lemmas.

Lemma 1. Let $W_{j}, 2 \leq j<n$, denote the state of the Markov chain from which the first transition to a state less than or equal to $j$ occurs. Then, for $r>j$,

$$
\mathrm{P}\left(T_{j}>0 \mid W_{j}=r\right) \geq \mathrm{P}\left(T_{j}>0 \mid W_{j}=j+1\right)=\mathrm{P}\left(D_{j+1}=1 \mid D_{j+1} \geq 1\right) .
$$

Proof. Let $Y_{r}=r-D_{r}$. Then,

$$
\begin{aligned}
\mathrm{P}\left(T_{j}>0 \mid W_{j}=r\right) & =\mathrm{P}\left(D_{r}=r-j \mid D_{r} \geq r-j\right) \\
& =\mathrm{P}\left(Y_{r}=j \mid Y_{r} \leq j\right) \\
& =\frac{\mathrm{P}\left(Y_{r}=j\right)}{\sum_{i=1}^{j} \mathrm{P}\left(Y_{r}=i\right)} \\
& =\frac{1}{\sum_{i=1}^{j} \mathrm{P}\left(Y_{r}=i\right) / \mathrm{P}\left(Y_{r}=j\right)} .
\end{aligned}
$$

But, for $i \leq j$, it follows from Corollary 4 that

$$
\frac{\mathrm{P}\left(Y_{r+1}=j\right)}{\mathrm{P}\left(Y_{r}=j\right)} \geq \frac{\mathrm{P}\left(Y_{r+1}=i\right)}{\mathrm{P}\left(Y_{r}=i\right)}
$$


or, equivalently, that

$$
\frac{\mathrm{P}\left(Y_{r+1}=i\right)}{\mathrm{P}\left(Y_{r+1}=j\right)} \leq \frac{\mathrm{P}\left(Y_{r}=i\right)}{\mathrm{P}\left(Y_{r}=j\right)} .
$$

Thus, by (4), $\mathrm{P}\left(T_{j}>0 \mid W_{j}=r\right)$ is nondecreasing in $r$.

Lemma 2. For all $j \geq 2$,

$$
\mathrm{P}\left(D_{j+1}=1 \mid D_{j+1} \geq 1\right) \geq \frac{\mathrm{e}^{-1}}{1-\mathrm{e}^{-1}} .
$$

Proof. Let $M_{k}=\sum_{i=0}^{k}(-1)^{i} / i$ !. By Proposition 1 we need to show that

$$
\frac{M_{j+1}}{1-(j+2) M_{j+2} /(j+1)} \geq \frac{\mathrm{e}^{-1}}{1-\mathrm{e}^{-1}} .
$$

That is, we need to show that, for all $n \geq 3$,

$$
M_{n}\left(1-\mathrm{e}^{-1}\right)-\mathrm{e}^{-1}\left(1-\frac{n+1}{n} M_{n+1}\right) \geq 0 .
$$

Case 1. Suppose that $n$ is even and that $n>2$. Then,

$$
\begin{aligned}
M_{n}(1 & \left.-\mathrm{e}^{-1}\right)-\mathrm{e}^{-1}\left(1-\frac{n+1}{n} M_{n+1}\right) \\
& =M_{n}\left(1-\mathrm{e}^{-1}\right)-\mathrm{e}^{-1}\left[1-\frac{n+1}{n}\left(M_{n}-\frac{1}{(n+1) !}\right)\right] \\
& =M_{n}\left(1+\frac{\mathrm{e}^{-1}}{n}\right)-\mathrm{e}^{-1}\left(1+\frac{1}{n n !}\right) \\
& \geq \mathrm{e}^{-1}\left(1+\frac{\mathrm{e}^{-1}}{n}\right)-\mathrm{e}^{-1}\left(1+\frac{1}{n n !}\right) \\
& =\frac{\mathrm{e}^{-1}}{n}\left(\mathrm{e}^{-1}-\frac{1}{n !}\right) \\
& >0
\end{aligned}
$$

where we used the fact that $M_{n}>\mathrm{e}^{-1}$.

Case 2. Suppose that $n$ is odd. In this case,

$$
\begin{aligned}
M_{n}(1 & \left.-\mathrm{e}^{-1}\right)-\mathrm{e}^{-1}\left(1-\frac{n+1}{n} M_{n+1}\right) \\
& =\left(M_{n+1}-\frac{1}{(n+1) !}\right)\left(1-\mathrm{e}^{-1}\right)-\mathrm{e}^{-1}\left(1-\frac{n+1}{n} M_{n+1}\right) \\
& =M_{n+1}\left(1+\frac{\mathrm{e}^{-1}}{n}\right)-\frac{1-\mathrm{e}^{-1}}{(n+1) !}-\mathrm{e}^{-1} \\
& \geq \mathrm{e}^{-1}\left(1+\frac{\mathrm{e}^{-1}}{n}\right)-\frac{1-\mathrm{e}^{-1}}{(n+1) !}-\mathrm{e}^{-1} \\
& =\mathrm{e}^{-1}\left(\frac{\mathrm{e}^{-1}}{n}+\frac{1}{(n+1) !}\right)-\frac{1}{(n+1) !},
\end{aligned}
$$


which will be nonnegative provided that

$$
\mathrm{e}^{-2} \geq \frac{n}{(n+1) !}\left(1-\mathrm{e}^{-1}\right)
$$

or, equivalently, that

$$
\mathrm{e}(\mathrm{e}-1) \leq \frac{(n+1) !}{n},
$$

which is easily seen to be true when $n \geq 3$. This completes the proof of Lemma 2 .

We need one additional lemma.

Lemma 3. As $n \rightarrow \infty, \mathrm{P}\left(D_{n}=0\right) \downarrow \mathrm{e}^{-1}$.

Proof. By Proposition 1,

$$
\mathrm{P}\left(D_{n}=0\right)=\frac{n+1}{n} M_{n+1},
$$

yielding $\lim _{n} \mathrm{P}\left(D_{n}=0\right)=\mathrm{e}^{-1}$. To show that the convergence is monotone, note that

$$
\begin{aligned}
\frac{n+1}{n} M_{n+1}-\frac{n+2}{n+1} M_{n+2} & =\frac{n+1}{n} M_{n+1}-\frac{n+2}{n+1}\left(M_{n+1}+\frac{(-1)^{n}}{(n+2) !}\right) \\
& =\frac{M_{n+1}}{n(n+1)}+\frac{(-1)^{n+1}}{(n+1)(n+1) !} .
\end{aligned}
$$

When $n$ is odd, the preceding is clearly positive. When $n$ is even, $M_{n+1}=M_{n}-1 /(n+1)$ !, and, thus, we must show that

$$
M_{n}-\frac{1}{(n+1) !} \geq \frac{1}{(n+1)(n-1) !}
$$

or, equivalently, that

$$
M_{n} \geq \frac{1}{n !}
$$

which follows since, for $n$ even,

$$
M_{n}=M_{n-1}+\frac{1}{n !} \geq \frac{1}{n !} .
$$

Proof of Proposition 5. Given that state $j$ is entered, the time spent in that state will have a geometric distribution with parameter $\mathrm{P}\left(D_{j}>0\right)$. Hence,

$$
\mathrm{E}\left[T_{j}\right]=\frac{\mathrm{P}\left(T_{j}>0\right)}{\mathrm{P}\left(D_{j}>0\right)} .
$$

Now, $\mathrm{P}\left(T_{n}>0\right)=1$, and, by Lemma $3, \mathrm{P}\left(D_{n}>0\right) \leq 1-\mathrm{e}^{-1}$, which verifies the first part of Proposition 5. Also, for $2 \leq j<n$, Lemmas 1 and 2 yield

$$
\mathrm{P}\left(T_{j}>0\right) \geq \mathrm{P}\left(D_{j+1}=1 \mid D_{j+1} \geq 1\right) \geq \frac{\mathrm{e}^{-1}}{1-\mathrm{e}^{-1}} .
$$

Hence, by Lemma 3,

$$
\mathrm{E}\left[T_{j}\right] \geq \frac{\mathrm{e}^{-1}}{\left(1-\mathrm{e}^{-1}\right)^{2}}=\frac{\mathrm{e}}{(\mathrm{e}-1)^{2}},
$$

which completes the proof of Proposition 5. 
TABLE 1.

\begin{tabular}{rrr}
\hline \multicolumn{1}{c}{$n$} & Lower bound & Upper bound \\
\hline 100 & 102.62 & 104.19 \\
1000 & 1004.72 & 1006.50 \\
1000000 & 1000011.08 & 1000013.41 \\
\hline
\end{tabular}

Corollary 4. We have

$$
n-1+\frac{\mathrm{e}}{n(\mathrm{e}-1)}+\frac{\mathrm{e}}{(\mathrm{e}-1)^{2}} \ln \left(\frac{n}{2}\right) \leq \mathrm{E}[N(n)] \leq n+\ln \left(\frac{2 n-1}{3}\right) .
$$

Proof. Let $X$ be uniformly distributed between $j-\frac{1}{2}$ and $j+\frac{1}{2}$. Then,

$$
\ln \left(\frac{j+1 / 2}{j-1 / 2}\right)=\int_{j-1 / 2}^{j+1 / 2} \frac{1}{x} \mathrm{~d} x=\mathrm{E}\left[\frac{1}{X}\right] \geq \frac{1}{\mathrm{E}[X]}=\frac{1}{j},
$$

where the inequality used Jensen's inequality. Hence,

$$
\sum_{j=2}^{n-1} \frac{1}{j} \leq \ln \left(\frac{n-1 / 2}{3 / 2}\right)=\ln \left(\frac{2 n-1}{3}\right)
$$

and the upper bound follows from Proposition 3. To obtain the lower bound, we use Proposition 4 along with the inequality

$$
\ln \left(\frac{j+1}{j}\right)=\int_{j}^{j+1} \frac{1}{x} \mathrm{~d} x \leq \frac{1}{j} .
$$

Remarks. 1 . Corollary 4 yields the results given in Table 1 .

2. It follows from Corollary 3 , using a coupling argument, that $N(n)$ is stochastically increasing in $n$.

\section{The circular case}

Whereas we have previously assumed that at each stage the clusters are randomly arranged in a linear order, in this section we suppose that they are randomly arranged around a circle, again with all possibilities being equally likely. We suppose that if a cluster ending with $i$ is immediately counterclockwise to a cluster beginning with $i+1$ then these clusters merge. Let $N^{*}(n)$ denote the number of stages needed until all $n$ elements are in a single cluster, and let $D_{n}^{*}$ denote the decrease in the number of clusters from state $n$.

Lemma 4. For $n \geq 2$,

$$
\mathrm{E}\left[D_{n}^{*}\right]=\operatorname{var}\left(D_{n}^{*}\right)=1 .
$$

Proof. If $B_{i}$ is the event that $i$ is the counterclockwise neighbor of $i+1$ then

$$
D_{n}^{*}=\sum_{i=1}^{n-1} \mathbf{1}_{B_{i}}
$$


Now,

$$
\mathrm{P}\left(B_{i}\right)=\frac{(n-2) !}{(n-1) !}=\frac{1}{n-1}, \quad i=1, \ldots, n-1,
$$

and, for $i \neq j$,

$$
\mathrm{P}\left(B_{i} B_{j}\right)=\frac{(n-3) !}{(n-1) !} .
$$

Hence,

$$
\mathrm{E}\left[D_{n}^{*}\right]=\sum_{i=1}^{n-1} \frac{1}{n-1}=1
$$

and

$$
\begin{aligned}
\operatorname{var}\left(D_{n}^{*}\right) & =\sum_{i=1}^{n-1} \frac{1}{n-1}\left(1-\frac{1}{n-1}\right)+2\left(\begin{array}{c}
n-1 \\
2
\end{array}\right)\left(\frac{(n-3) !}{(n-1) !}-\frac{1}{(n-1)^{2}}\right) \\
& =\frac{n-2}{n-1}+1-\frac{n-2}{n-1} \\
& =1 .
\end{aligned}
$$

Proposition 6. We have

$$
\mathrm{E}\left[N^{*}(n)\right]=n-1 .
$$

Proof. The proof is by induction on $n$. Because $\mathrm{P}\left(N^{*}(2)=1\right)=1$, it is true when $n=2$, and so assume that $\mathrm{E}\left[N_{k}^{*}\right]=k-1$ for all $k=2, \ldots, n-1$. Then,

$$
\mathrm{E}\left[N^{*}(n) \mid D_{n}^{*}\right]=1+\mathrm{E}\left[N^{*}\left(n-D_{n}^{*}\right) \mid D_{n}^{*}\right]
$$

yielding

$$
\begin{aligned}
\mathrm{E}\left[N^{*}(n)\right] & =1+\sum_{i=0}^{n-1} \mathrm{E}\left[N^{*}(n-i)\right] \mathrm{P}\left(D_{n}^{*}=i\right) \\
& =1+\mathrm{E}\left[N^{*}(n)\right] \mathrm{P}\left(D_{n}^{*}=0\right)+\sum_{i=1}^{n-1} \mathrm{E}\left[N^{*}(n-i)\right] \mathrm{P}\left(D_{n}^{*}=i\right) \\
& =1+\mathrm{E}\left[N^{*}(n)\right] \mathrm{P}\left(D_{n}^{*}=0\right)+\sum_{i=1}^{n-1}(n-i-1) \mathrm{P}\left(D_{n}^{*}=i\right) \\
& =1+\mathrm{E}\left[N^{*}(n)\right] \mathrm{P}\left(D_{n}^{*}=0\right)+(n-1)\left(1-\mathrm{P}\left(D_{n}^{*}=0\right)\right)-\mathrm{E}\left[D_{n}^{*}\right] \\
& =1+\mathrm{E}\left[N^{*}(n)\right] \mathrm{P}\left(D_{n}^{*}=0\right)+(n-1)\left(1-\mathrm{P}\left(D_{n}^{*}=0\right)\right)-1,
\end{aligned}
$$

which proves the result.

Remark. Proposition 6 could also have been proved by using a martingale stopping argument, as in the proof of Proposition 4.

Proposition 7. For $n>2$,

$$
\operatorname{var}\left(N^{*}(n)\right)=n-1
$$


Proof. Let $V(n)=\operatorname{var}\left(N^{*}(n)\right)$. The proof is by induction on $n$. As it is true for $n=3$, since $N^{*}(3)$ is geometric with parameter $\frac{1}{2}$, assume it is true for all values between 2 and $n$. Now,

$$
\operatorname{var}\left(N^{*}(n) \mid D_{n}^{*}\right)=\operatorname{var}\left(N^{*}\left(n-D_{n}^{*}\right) \mid D_{n}^{*}\right)
$$

and, from (5) and Proposition 6,

$$
\mathrm{E}\left[N^{*}(n) \mid D_{n}^{*}\right]=n-D_{n}^{*} .
$$

Hence, by the conditional variance formula,

$$
\begin{aligned}
V(n) & =\sum_{i=0}^{n-1} V(n-i) \mathrm{P}\left(D_{n}^{*}=i\right)+\operatorname{var}\left(D_{n}^{*}\right) \\
& =V(n) \mathrm{P}\left(D_{n}^{*}=0\right)+\sum_{i=1}^{n-1} V(n-i) \mathrm{P}\left(D_{n}^{*}=i\right)+1 .
\end{aligned}
$$

Now, because $\mathrm{P}\left(D_{n}^{*}=n-2\right)=0$ and $V(1)=0$, the induction hypothesis yields

$$
\sum_{i=1}^{n-1} V(n-i) \mathrm{P}\left(D_{n}^{*}=i\right)=\sum_{i=1}^{n-1}(n-i-1) \mathrm{P}\left(D_{n}^{*}=i\right) .
$$

Hence, from (6),

$$
\begin{aligned}
V(n) & =V(n) \mathrm{P}\left(D_{n}^{*}=0\right)+(n-1)\left(1-\mathrm{P}\left(D_{n}^{*}=0\right)\right)-\mathrm{E}\left[D_{n}^{*}\right]+1 \\
& =V(n) \mathrm{P}\left(D_{n}^{*}=0\right)+(n-1)\left(1-\mathrm{P}\left(D_{n}^{*}=0\right)\right),
\end{aligned}
$$

which proves the result.

\section{References}

[1] Arratia, R. and Tavaré, S. (1992). The cycle structure of random permutations. Ann. Prob. 20, 1567-1591.

[2] De Montmort, P. R. (1708). Essay d'Analyse sur le Jeux de Hazard. Quillau, Paris.

[3] Diaconis, P., Fulman, J. and Guralnick, R. (2008). On fixed points of permutations. J. Algebraic Combin. 28, 189-218.

[4] Feller, W. (1968). An Introduction to Probability Theory and Its Applications, Vol. I, 3rd edn. John Wiley, New York.

[5] RaO, M. B. and Kasala, S. (2008). A discrete probability problem in chemical bonding. Preprint, Universite de Technologie de Compiegne, France.

[6] Ross, S. M. And Peköz, E. (2007). A Second Course in Probability. ProbabilityBookstore.com, Boston, MA. 